\title{
Aumento de las infestaciones por Demodex sp. durante el periodo del primer confinamiento marzo a noviembre 2020 , durante la pandemia por SARS-CoV-2
}

\author{
Increase in Demodex sp infestations during the \\ first confinement period, March to November \\ 2020, during the SARS-CoV-2 pandemic
}

\author{
María Emilia Del Pino Flores, ${ }^{*}$ Mariana López Garza, ${ }^{\ddagger}$ Pedro Orozco del Pino ${ }^{\S}$ \\ Citar como: Del Pino FME, López GM, Orozco PP. Aumento de las infestaciones por Demodex sp. durante \\ el periodo del primer confinamiento marzo a noviembre 2020, durante la pandemia por SARS-CoV-2. \\ Acta Med Grupo Angeles. 2021; 19 (s1): s76-s79. https://dx.doi.org/10.35366/101033
}

\section{Resumen}

La Demodecidosis es una parasitosis de la piel asociada a enfermedades donde se afecta la unidad pilosebácea como rosácea, dermatitis seborreica, Pitiriasis folliculorum, foliculitis y blefaritis. Se observaron pacientes con erupciones acneiformes, acné activo y rosácea desde marzo a noviembre del 2020 y realizamos biopsia con cianoacrilato considerándose positivas con más de cinco Demodex por $\mathrm{cm}^{2}$. Los pacientes de marzo (no confinados) actuaron como control y los de abril a noviembre (confinados) como objeto de estudio. Se cuantificaron los casos y se realizó análisis estadístico. Se estudiaron 743 pacientes, 147 presentaron manifestaciones clínicas recientes de erupciones acneiformes, acné activo y rosácea, 145 biopsias resultaron positivas, la edad promedio fue de 28 años (DS: +/-13, rango:11-71). Los casos positivos aumentaron durante el confinamiento del $7 \%$ en marzo al $18 \%$ en abril y posteriormente a cifras entre 18 y $35 \%$, el análisis estadístico resultó positivo a partir del segundo mes de confinamiento y se mantuvo significativo por 6 meses. Conclusiones: El confinamiento incrementó los casos de demodex y la infección fue tan sintomática que obligó a los pacientes a acudir al médico sin importar el riesgo de la pandemia.

Palabras clave: Demodex, demodecidosis,acné, covid, rosacea, dermatitis seborreica.
Abstract

Demodicosis is a skin parasitosis associated with diseases that affect the pilosebaceous unit such as Rosacea, Seborrheic Dermatitis, Pityriasis folliculorum, Folliculitis and Blepharitis. Patients with acneiform eruptions, active acne and rosacea were observed from March to November 2020 and we performed a cyanoacrylate biopsy, considering them positive with more than five Demodex per $\mathrm{cm}^{2}$. Patients from March (unconfined) acted as controls and those from April to November (confined) as the object of study. Cases were quantified and statistical analysis was performed. 743 patients were studied, 147 presented recent clinical manifestations of acneiform eruptions, active acne and rosacea, 145 biopsies were positive, the average age was 28 years (DS: 13-r: 11-71). Positive cases increased during confinement from $7 \%$ in March to $18 \%$ in April and subsequently to figures between 18 and $35 \%$, the statistical analysis was positive from the second month of confinement and remained significant for 6 months. Conclusions: The confinement increased the cases of demodex and the infection was so symptomatic that it forced patients to go to the doctor regardless of the risk of the pandemic.

Keywords: Demodex, demodecosis, acne, covid, rosacea, seborrheic dermatitis.
* Dermatóloga. Hospital Ángeles Pedregal.

‡ Residente de Pediatría. Hospital Ángeles Pedregal.

§ Asistente de Investigación. Departamento de Bioestadística, Universidad de Michigan.
Correspondencia:

María Emilia Del Pino Flores

Correo electrónico: delpinoflores@gmail.com

www.medigraphic.com/actamedica 


\section{INTRODUCCIÓN}

Los ácaros de Demodex sp., de la familia Demodicidae, suborden trombidiformes, orden acariformes, son ácaros minúsculos, descritos por primera vez por Henle y Berger en $1841,{ }^{1}$ se consideran microorganismos comensales de la microfauna normal de la piel de los mamíferos, donde residen de forma permanente dentro o fuera del folículo piloso y glándulas sebáceas. Los Demodex folliculorum y Demodex brevis se consideran propios de los humanos y la Demodecidosis se ha reportado como una infección de la piel humana causada por estos ectoparásitos que han sido relacionados con diversas condiciones clínicas, en donde la unidad pilosebácea está afectada como en rosácea, dermatitis seborreica, Pitiriasis folliculorum, foliculitis y blefaritis. ${ }^{1,2}$ El mecanismo de transmisión es por el contacto piel con piel, por lo que resulta importante en esta pandemia debido al hacinamiento y el contacto estrecho y prolongado entre las personas.

De las dos especies de Demodex descritas en la piel humana, $D$. folliculorum y $D$. brevis, la primera es la más larga con una longitud de 0.3 a $0.4 \mathrm{~mm},{ }^{2}$ y habita en el infundíbulo del folículo piloso, mientras que $D$. brevis tiene de 0.2 a $0.3 \mathrm{~mm}$ de largo, ${ }^{2}$ se encuentran típicamente en las glándulas sebáceas y de Meibomio, ambos se localizan preferentemente en la región facial, afectando especialmente la frente, mejillas y región naso labial. La anatomía del ácaro consiste en tres partes principales, un gnathostoma (boca), podosoma (tórax) y opistosoma (abdomen), que se encuentran cubiertos por un exoesqueleto, se alimentan del sebo y las células epiteliales y viven aproximadamente entre 14 y 18 días en la piel humana. ${ }^{1,2}$

La prevalencia de Demodex en humanos saludables es difícil de estimar, teniendo rangos de entre 17 a $72 \% .^{3}$ Se reporta que el porcentaje de individuos infestados incrementa con la edad, con una prevalencia de $95 \%$ en los adultos mayores de 71 años y llegando a ser de 100\% en los individuos de 96 años o más, presenta un aumento importante entre la cuarta y sexta década de la vida con un 69 a $87 \%$ de prevalencia, mientras que en los adultos jóvenes de 19 a 25 años sigue siendo mayor que en los menores de 15 años, con rangos de entre 14 a $34 \%$ y 5.88 a $13 \%$ respectivamente. ${ }^{4,5}$

No existe una metodología específica para localizar al acaro en la piel humana, la mayoría de los estudios se basan en distintos métodos de observación, se pueden utilizar los métodos de la cinta de celofán, raspados de la piel, inspección por microscopia de muestras del sebo de la piel y la biopsia de la superficie de la piel, siendo este último el más común, ya que muestra una mayor sensibilidad para medir la densidad del ácaro presente en la piel. El diagnóstico se realiza con la presencia de más de cinco ácaros por centímetro cuadrado. ${ }^{3,5-7}$
El modo de transmisión del Demodex puede empezar desde el nacimiento, de madre a hijo, o a través del contacto piel a piel con otras personas en el transcurso de la vida, lo que explica el aumento de la prevalencia con la edad, aunado a los cambios en las características del sebo de la piel y en el sistema inmune de las personas. El compromiso inmunológico, cualquiera que sea la causa, está relacionado con el aumento en el número de $D$. folliculorum presentes en la piel. ${ }^{6}$

La demodicosis, un término que agrupa las enfermedades cutáneas causadas por ácaros del género Demodex, descrita por primera vez en 1961 como una enfermedad sui géneris de la unidad pilosebácea asociada a Demodex, se divide en primaria y secundaria, esta última asociada a inmunosupresión. Las manifestaciones clínicas de la variedad primaria incluyen eritema, pápulas, pústulas, descamación, sequedad folicular y en ocasiones prurito. ${ }^{6,8}$ Siempre que este tipo de sintomatología esté presente sin antecedentes de lesiones como acné, rosácea o dermatitis perioral preexistente, no responda a la terapia estándar y no estén implicados patógenos bacterianos, se deberá considerar la presencia del ácaro, donde para confirmar el diagnóstico tendrá que encontrarse un incremento anormal en la colonización por Demodex en las lesiones activas y remitir con el tratamiento adecuado con acaricidas tópicos o sistémicos. $3,7,9$

El tratamiento recomendado para la demodicosis incluye crema de crotamitón, tetraciclinas y metronidazol tópico o sistémico, además de ivermectina tópica y oral. ${ }^{3,7} \mathrm{El}$ metronidazol y la ivermectina han demostrado una alta eficacia cuando se administran juntos, mostrando en $71.6 \%$ de los pacientes remisión completa en lesiones de rosácea y blefaritis, en comparación con el esquema de ivermectina que mostró remisión completa en el $45 \%$ de los casos. ${ }^{10}$

D. folliculorum está asociado con eritema y descamación epitelial, mientras que $D$. brevis es más común con erupción papulopustular, descamación simétrica y eritema, que pueden predisponer a enfermedades tipo rosácea. Desde 1930 se relacionó la presencia del ácaro en la piel con el desarrollo de diversas enfermedades cutáneas, como Pitiriasis folliculorum, que se caracteriza por pequeñas pápulas con escama fina, eritema y prurito, rosácea, mucinosis folicular y demodesidosis. ${ }^{2}$ Los mecanismos causales de la relación del ácaro Demodex con la presencia de las lesiones en la piel son el bloqueo mecánico del folículo piloso por parte del parásito, produciendo distención y formación de hiperqueratosis en las paredes del folículo, esto favorece la formación de respuesta inflamatoria con granulomas por cuerpo extraño e hiperestimulación del sistema inmune, lo que produce una reacción de hipersensibilidad. En la rosácea se ha demostrado una relación con la presencia de altos recuentos de Demodex en la piel, entre 90 y 100\%, los posibles vectores de estas lesiones son los microorganis- 
mos saprófitos más la presencia de bacterias como Bacillus oleronius; otros factores de riesgo asociados al aumento de ácaros en la piel son la exposición a mascotas (gatos, perros), seborreo y el uso de esteroides tópicos. ${ }^{11-13}$

Los diagnósticos diferenciales en los pacientes que acuden por lesiones faciales inflamatorias son el acné y rosácea que empezaron o se agravaron durante los meses de confinamiento por la pandemia SARS-CoV-2 de marzo a noviembre 2020 .

\section{MATERIAL Y MÉTODOS}

Estudio observacional y descriptivo, donde se incluyeron todos los pacientes con manifestaciones clínicas recientes de erupciones acneiformes, acné activo y rosácea que acudieron a consulta durante el periodo de marzo a noviembre del 2020. A los pacientes que presentaban afección de la cara con prurito, eritema, descamación, comedones, pápulas y pústulas con o sin seborrea, se les realiza biopsia con cianoacrilato de rutina. La biopsia fue de la superficie de la piel, en la parte central y en una mejilla, y consiste en la colocación de una gota de cianoacrilato en la piel elegida, se pone en contacto con un portaobjetos de vidrio durante 10 segundos, abarcando una superficie estándar de $1 \mathrm{~cm}^{2}$, después se retira con cuidado, obteniendo una muestra de capa córnea y folículos, la cual fue observada bajo el microscopio de luz. Se tomaron como muestra positiva las que mostraron más de cinco Demodex por $\mathrm{cm}^{2}$.

Consideramos los casos del mes de marzo como pacientes que no estuvieron en confinamiento, los de abril a noviembre como pacientes en confinamiento. Presentamos intervalos de 95\% de confianza del porcentaje de pacientes con Demodex de cada mes. Se realiza una prueba de hipótesis de comparación de proporciones entre cada mes de confinamiento contra marzo a un nivel de $99.4 \%$ de confianza, el cual se eligió para tomar en cuenta que se están haciendo ocho pruebas estadísticas con el mes de marzo. El porcentaje de pacientes con Demodex fue contemplado para poder comparar meses con afluencia en consulta distinta.

\section{RESULTADOS}

Se estudiaron 743 pacientes, de éstos, 147 presentaron manifestaciones clínicas recientes de erupciones acneiformes, acné activo y rosácea, y a todos se les realizó biopsia con cianoacrilato, 145 resultaron positivos a Demodex sp., la edad promedio fue de 28 años (DS: \pm 13 , rango:11-71).

El número de pacientes totales por mes, número de días de consulta, porcentaje de casos positivos a Demodex sp., el cambio en el porcentaje de Demodex con el mes de marzo y una columna que indica si la diferencia es estadísticamente significativa se muestran en la Tabla 1. Los meses de abril y mayo no muestran un aumento significativo en el porcentaje de pacientes con Demodex $s p .$, los meses de junio, julio, agosto, septiembre, octubre y noviembre sí muestran un aumento significativo en el porcentaje de pacientes con Demodex sp. a un nivel de 99.4\% de confianza.

Las entidades diagnosticadas en los pacientes con Demodex sp. positivo se mencionan en la Tabla 2.

\section{DISCUSIÓN}

La pandemia provocó múltiples cambios en las costumbres de la vida diaria, el confinamiento, la cancelación de las

\begin{tabular}{|c|c|c|c|c|c|c|}
\hline & $\begin{array}{l}\text { Número total } \\
\text { de consultas }\end{array}$ & Días & $\begin{array}{l}\text { Demodex } \\
\text { positivos }\end{array}$ & $\begin{array}{l}\text { \% Demodex } \\
\text { positivo }\end{array}$ & $\begin{array}{c}\text { Cambio respecto } \\
\text { a marzo }\end{array}$ & $\begin{array}{l}\text { Resultado de la } \\
\text { prueba estadística }\end{array}$ \\
\hline Marzo & 76 & 12 & 7 & 9.2 & 0.0 & - \\
\hline Abril & 31 & 15 & 7 & 18.9 & 9.7 & No \\
\hline Mayo & 45 & 17 & 13 & 28.8 & 19.6 & No \\
\hline Junio & 51 & 17 & 18 & 35 & 25.8 & Sí \\
\hline Julio & 118 & 19 & 42 & 35.5 & 26.3 & Sí \\
\hline Agosto & 90 & 16 & 32 & 35 & 25.8 & Sí \\
\hline Septiembre & 116 & 17 & 34 & 29 & 19.8 & Sí \\
\hline Octubre & 127 & 16 & 44 & 34 & 24.8 & Sí \\
\hline Noviembre & 108 & 16 & 31 & 28 & 18.8 & Sí \\
\hline
\end{tabular}


Tabla 2: Entidades clínicas diagnosticadas en pacientes con Demodex sp. positivo.

\begin{tabular}{lc} 
Diagnóstico & Número \\
\hline Sólo demodecidosis & $33(22.1 \%)$ \\
Acné & $48(33 \%)$ \\
Dermatitis seborreica & $21(14.5 \%)$ \\
Rosácea & $8(5.5 \%)$ \\
Erupción acneiforme & 6 \\
Foliculitis barba & 4 \\
Tiña del cuerpo & 4 \\
Acné mask & 2 \\
Blefaritis & 2 \\
Otros & 8
\end{tabular}

salidas y el aumento de actividades laborales y educativas desde casa mostraron repercusiones.

El confinamiento provocó una disminución de la consulta médica, que llegó a ser de alrededor de 80\% en abril, mayo y junio, iniciando un aumento de la consulta que coincide con el cambio de color del semáforo epidemiológico; sin embargo, el porcentaje de casos de Demodex sp. en la consulta se incrementa en forma absoluta, pasando de $9.7 \%$ en marzo a estar por encima de $19 \%$ a partir de mayo, esta tendencia se mantuvo con variaciones hasta el mes de noviembre. Tomemos en cuenta que los pacientes de principio de abril tendrían muy poco confinamiento a comparación de aquéllos del final de abril. Creemos que eso puede explicar por qué el porcentaje de pacientes en abril es intermedio entre marzo y los demás meses. El Demodex sp. aislado se considera un patógeno y se presentó en 30 casos (23\%), la presentación más común fue acné en en 48 pacientes (33\%), la dermatitis seborreica fue la tercera asociación más frecuente con 21 casos (15\%); Ilama la atención que el hallazgo más comúnmente reportado en la literatura que es la asociación de Demodex con Rosacea solo se presentó en 8 pacientes (5.5\%), quizá porque todos estos casos tenían poco tiempo de evolución. 3,7,9

El confinamiento disminuyó las actividades al aire libre, modificó las conductas higiénicas, aumentó el hacinamiento y estas fueron las causas probables del incremento de la infestación, aunadas al aumento de la ansiedad por la pandemia y el confinamiento.

Este trabajo es limitado ya que sólo abarca un consultorio privado, pero debido al relajamiento de las normas de confinamiento es difícil replicarlo. Creemos que los médicos debemos estar atentos para detectar y manejar estas nuevas entidades que surgen en respuesta a las nuevas condiciones de vida.

\section{CONCLUSIONES}

A pesar de la disminución en el número de casos que asistieron a consulta médica, los síntomas en la piel fueron para ellos tan importantes que corrieron el riesgo de salir a buscar consejo médico a pesar del riesgo de contagio al virus SARS-CoV-2.

\section{REFERENCIAS}

1. Foley R, Kelly P, Gatault S, Powell F. Demodex: a skin resident in man and his best friend. JEADV. 2020. doi: 10.1111/jdv.16461.

2. Thoemmes MS, Fergus J, Urban J et al. Ubiquity and diversity of human-associated Demodex. PLoS ONE. 2014; 9 (8): e106265.

3. Bachmeyer C. Moreno-Sabater A. Demodex foliculitis. CMAJ. 2017; 189 (25): E865.

4. Elston CA, Elston DM. Demodex mites. Clin Dermatol. 2014; 32 (6): 739-743.

5. Litwin D. Chen W. Dzika E. Korycinska J. Human permanent ectoparasites; recent advances on biology and clinical significance of Demodex mites: narrative review article. Iran J Parasitol. 2017; 12 (1): 12-21.

6. Gazi U, Gureser AS, Oztekin A, Karasartova D, Kosar-Acar N, Derici MK et al. Skin-homing T-cell responses associated with Demodex infestation and rosacea. Parasite Immunol. 2019; 41 (8): e12658.

7. Olivares JJ, Cherit JD, Tomoka MTH, González JMD. Demodecidosis: una revisión clínica y terapéutica. Dermatologia CMQ. 2014; 12 (2): 122-127.

8. Aumond S. Bittron E. Palpebral and facial skin infestation by Demodex folliculorum. Cont Lens Anterior Eye. 2020; 43 (2): 115-122.

9. Chen W. Plewing G. Human demodicosis: revisit and a proposed classification. Br J Dermatol. 2014; 170 (6): 1219-1225.

10. Salem DA, El-Shazly A, Nabih N, El-Bayoumy Y, Saleh S. Evaluation of the efficacy of oral ivermectin in comparison with ivermectinmetronidazole combined therapy in the treatment of ocular and skin lesions of Demodex folliculorum. Int J Infect Dis. 2013; 17 (5): 343-347.

11. Castellanos LH, Pardo CG. Rosácea, Demodex folliculorum y metronidazol tópico. Rev Fac Med UN Col. 1998; 46 (4): 194-198.

12. Jarmuda S, O'Reilly N, Zaba R, Jakubowicz O, Szkaradkiewicz A, Kavanagh K. Demodex y bacteria en la rosacea. J Med Microbiol. 2012; 61: 1504-1510.

13. Trejo ME, Puell RL, Salomón M, Vidal AM, Del Solar CM, Bravo PF et al. Demodecidosis en pacientes con rosácea. Rev Med Hered. 2007; 18 (1): 15-21.

Conflicto de intereses: Los autores no recibieron ningún tipo de financiamiento ni tienen conflicto de intereses. 